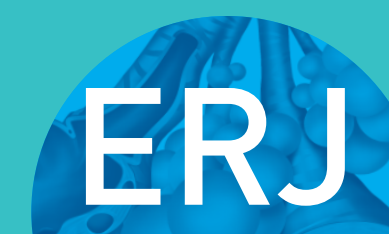

open research
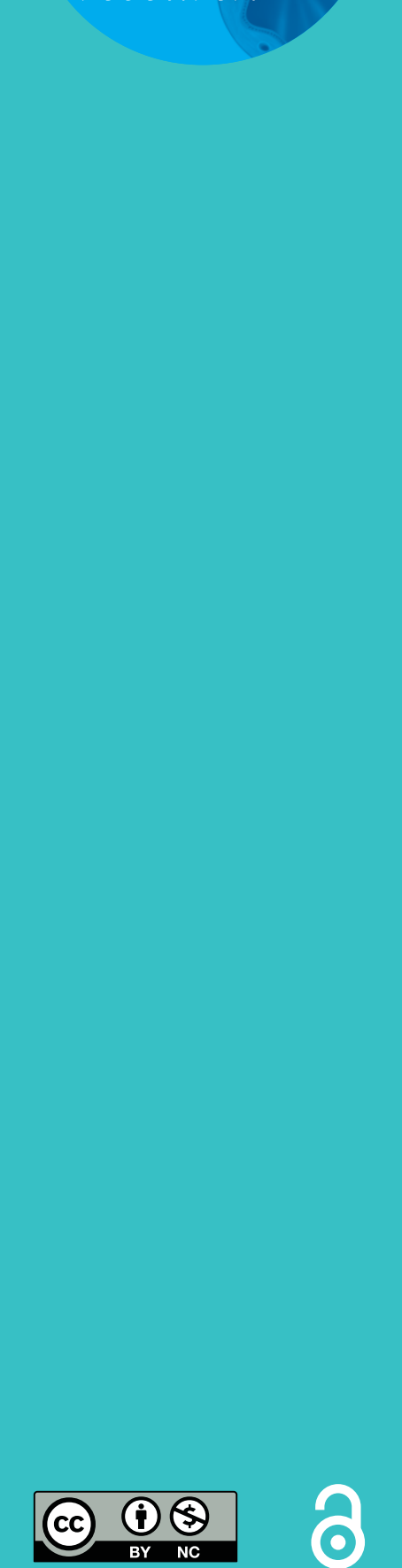

\section{Aspergillus-induced superoxide production by cystic fibrosis phagocytes is associated with disease severity}

\author{
Shan F. Brunel ${ }^{1}$, Janet A. Willment (1) ${ }^{1}$, Gordon D. Brown, Graham Devereux ${ }^{2}$ \\ and Adilia Warris ${ }^{1}$
}

Affiliations: ${ }^{1}$ Aberdeen Fungal Group, MRC Centre for Medical Mycology, Institute of Medical Sciences, University of Aberdeen, Aberdeen, UK. ${ }^{2}$ Cystic Fibrosis Clinic, Aberdeen Royal Infirmary, Aberdeen, UK.

Correspondence: A. Warris, Aberdeen Fungal Group, MRC Centre for Medical Mycology, Rm 4.23, Institute of Medical Sciences, Foresterhill, Aberdeen, AB25 2ZD, UK. E-mail: a.warrisdabdn.ac.uk

ABSTRACT Aspergillus fumigatus infects up to 50\% of cystic fibrosis (CF) patients and may play a role in progressive lung disease. As cystic fibrosis transmembrane conductance regulator is expressed in cells of the innate immune system, we hypothesised that impaired antifungal immune responses play a role in CF-related Aspergillus lung disease.

Peripheral blood mononuclear cells, polymorphonuclear cells (PMN) and monocytes were isolated from blood samples taken from CF patients and healthy volunteers. Live-cell imaging and colorimetric assays were used to assess antifungal activity in vitro. Production of reactive oxygen species (ROS) was measured using luminol-induced chemiluminescence and was related to clinical metrics as collected by case report forms.

CF phagocytes are as effective as those from healthy controls with regards to phagocytosis, killing and restricting germination of A. fumigatus conidia. ROS production by CF phagocytes was up to four-fold greater than healthy controls $(\mathrm{p}<0.05)$. This effect could not be replicated in healthy phagocytes by priming with lipopolysaccharide or serum from CF donors. Increased production of ROS against A. fumigatus by $\mathrm{CF}$ PMN was associated with an increased number of clinical exacerbations in the previous year $(\mathrm{p}=0.007)$ and reduced lung function (by forced expiratory volume in $1 \mathrm{~s})(\mathrm{p}=0.014)$.

CF phagocytes mount an intrinsic exaggerated release of ROS upon A. fumigatus stimulation which is associated with clinical disease severity.

@ERSpublications

Excessive superoxide production by CF phagocytes against A. fumigatus is associated with clinical disease severity http://ow.ly/Elwy30i8mLe

Cite this article as: Brunel SF, Willment JA, Brown GD, et al. Aspergillus-induced superoxide production by cystic fibrosis phagocytes is associated with disease severity. ERJ Open Res 2018; 4: 00068-2017 [https://doi.org/10.1183/23120541.00068-2017].

This article has supplementary material available from openres.ersjournals.com

Received: June 032017 | Accepted after revision: Jan 262018

Copyright $\odot$ ERS 2018. This article is open access and distributed under the terms of the Creative Commons Attribution Non-Commercial Licence 4.0. 


\section{Introduction}

Cystic fibrosis (CF) is the most common life shortening inherited disease among Caucasians with an estimated prevalence of 0.74 per 10000 in Europe [1]. Mutations in the gene encoding the cystic fibrosis transmembrane conductance regulator (CFTR) in epithelial cells affect mucus fluid dynamics and immune function, which promotes pathogen survival and lung inflammation [2]. CFTR expression has also been demonstrated in cells of the innate and adaptive immune system [3,4] and is critical for normal function [5]. In vitro studies have demonstrated that $\mathrm{CF}$ monocytes and neutrophils display reduced killing activity against Pseudomonas aeruginosa [6]. The underlying molecular mechanisms appear to be phagocyte specific and include impaired chloride transport, abnormal complement activation and defective autophagy [6-8]. Reduced killing of Burkholderia cepacia by CF macrophages has also been reported and linked to defective autophagy [9].

To date, exploration of the innate immune response of CF phagocytes has focussed on typical CF bacterial pathogens, such as $P$. aeruginosa and $B$. cepacia [6-9]. More recently, however, it has been suggested that the ubiquitous environmental fungus Aspergillus fumigatus may play a critical role in CF lung disease. A. fumigatus can cause a wide range of disease as a function of the host response. Pulmonary aspergillosis ranges from angio-invasive disease with a high fungal burden in patients with a profoundly weakened immune system, to allergic disease with exaggerated inflammatory responses damaging the host tissue as seen in allergic bronchopulmonary aspergillosis (ABPA) [10]. It has been reported that up to $58 \%$ of $\mathrm{CF}$ patients are colonised with A. fumigatus [11] and that an estimated $47.7 \%$ of CF adults are affected by either ABPA or Aspergillus infection [12]. In addition, persistent A. fumigatus infection in CF is adversely correlated with lung function and hospitalisation [13, 14]. The aim of the current study was to explore the antifungal defence mechanisms of CF phagocytes, to compare them with those of healthy controls and to relate them to clinical metrics of CF disease severity.

\section{Material and methods}

A single centre cross-sectional study was conducted. Additional details on the methods are provided in the supplementary material.

\section{Participants}

Patients attending the adult CF clinic at Aberdeen Royal Infirmary (Aberdeen, UK) were invited to participate. The inclusion criteria were: CF-related lung disease, aged $\geqslant 18$ years, stable clinical condition for $>4$ weeks. Lung-transplant recipients were excluded as well as those on steroids or other anti-inflammatory therapy. Healthy control participants were drawn from the staff working in the Institute of Medical Sciences (University of Aberdeen). The study was approved by the North of Scotland Research Ethics Service (14/NS/1072) and all participants provided written informed consent. Participants were asked to provide a venous blood sample (minimum $10 \mathrm{~mL}$ and maximum $40 \mathrm{~mL}$ ) using standard procedures. Clinical data from each participating CF patient was collected and included: CF genotype, forced expiratory volume in $1 \mathrm{~s}$ (FEV1), body mass index (BMI), age, CF-related diabetes, Aspergillus IgG and IgE, days in hospital, pulmonary exacerbations, Ivacaftor treatment, sputum culture results and antimicrobials prescribed over the previous year.

\section{Human peripheral blood phagocytes}

Peripheral blood mononuclear cell (PBMC) and polymorphonuclear cell (PMN) fractions were isolated from EDTA blood samples by density gradient centrifugation. Monocytes (MNC) were purified from the PBMC fraction using CD14+ microbeads (Miltenyi Biotec, UK) according to the manufacturer's protocol

\section{Microorganisms}

Conidia of the V45-07 (clinical isolate) and dsRed A293-A. fumigatus strains of A. fumigatus were used. The lab reference strain of $P$. aeruginosa was PA01 (see supplementary material).

\section{Live-cell video microscopy}

The dsRed A293-A. fumigatus was stained with AF633 (Fluorescent Aspergillus Reporter (FLARE) conidia) as previously described [15]. To an eight well glass-bottomed microslide was added PMN or MNC $\left(1.2 \times 10^{5}\right)$, which was then stimulated with FLARE conidia $\left(3.6 \times 10^{5}\right)$. Imaging was performed with a spinning disk confocal microscope. 
or PMN $\left(1 \times 10^{5}\right)$. Subsequently, A. fumigatus conidia $\left(1 \times 10^{5}\right)$ was added and the whole incubated for $18 \mathrm{~h}$. Cells were lysed, incubated for $2 \mathrm{~h}$ with XTT-Menadione solution and absorbance was measured.

\section{Oxidative burst}

Oxygen radical production was evaluated by luminol-based chemiluminescence as previously published [16]. PMN, PBMC or MNC $\left(5 \times 10^{5}\right)$ were incubated with A. fumigatus conidia $\left(5 \times 10^{7}\right.$ per $\left.\mathrm{mL}\right)$, latex beads $\left(5 \times 10^{7}\right.$ per $\left.\mathrm{mL}\right)$, depleted zymosan $\left(200 \mu \mathrm{g} \cdot \mathrm{mL}^{-1}\right)$, lipopolysaccharide (LPS; $\left.50 \mathrm{ng} \cdot \mathrm{mL}^{-1}\right)$ or P. aeruginosa $\left(5 \times 10^{7}\right.$ per $\left.\mathrm{mL}\right)$.

\section{Statistical considerations}

Nonparametric tests (Mann-Whitney, Spearman's rank correlation coefficient (Spearman's rho), Jonckheere-Tepestra) were used for all analyses using IBM SPSS Statistics v24.0 (IBM Corp, Armonk, NY, USA) and GraphPad Prism v5.04 (GraphPad Software, La Jolla, CA, USA). Reactive oxygen species (ROS) production over time from $\mathrm{CF}$ and control cells was analysed using repeated measures ANOVA with Greenhouse-Geisser correction.

\section{Results}

Clinical characteristics of participants

Of the 60 eligible patients attending the clinic, $30 \mathrm{CF}$ patients participated in the study (median age 32 years (range 18-67 years); 69\% male). All of the participants with CF were either homozygous or heterozygous for the Phe508del mutation. Median BMI was 22.3 (range 18.3-29) and median FEV1 was $50 \%$ predicted (range $26-92 \%$ predicted). Three patients received Ivacaftor, 17 received anti-Pseudomonas inhalation antibiotics, and one received antifungal therapy. Sputum grew A. fumigatus and P. aeruginosa in $36 \%$ and $72 \%$ of the patients, respectively. Healthy participants were between 18 and 60 years of age.

\section{Phagocytic and antifungal activity of cystic fibrosis phagocytes}

PMN and MNC from CF and control subjects were co-incubated with A. fumigatus conidia and uptake of conidia was imaged with live-cell video microscopy. No differences were observed between $\mathrm{CF}$ and healthy phagocytes in either the proportion of cells containing conidia (figure la) or in the number of conidia contained within each cell. To determine the ability of phagocytes to kill A. fumigatus, live-cell imaging using FLARE conidia was performed. FLARE conidia were not killed within a 6-h incubation period with either neutrophils or monocytes for both healthy and CF donors (data not shown). No differences were observed in the proportion of germinating conidia when co-incubated with either PMN or MNC at any of the measured time points (figure 1b). XTT colorimetric assay demonstrated that CF PBMC had a significantly higher mean fungicidal activity $(78.6 \% \pm 3.8 \%)$ compared to healthy PBMC $(61.5 \% \pm 2.3 \%)(\mathrm{p}=0.002)$. No significant differences were observed in the fungicidal activity of PMN (45\% $\pm 12 \%$ versus $41.5 \% \pm 6.4 \%)$ or MNC (65.7\% $\pm 2.9 \%$ versus $64.4 \% \pm 4.6 \%)$ from CF and healthy controls, respectively (figure 1c).

\section{Reactive oxygen species production by cystic fibrosis phagocytes}

ROS production after incubation with $A$. fumigatus conidia was significantly increased by CF PMN, PBMC and MNC when compared to healthy controls (figures 2a-d). CF PMN, PBMC and MNC showed a three- to four-fold increase in total ROS production over $2 \mathrm{~h}$ of stimulation compared to healthy cells ( $\mathrm{p}=0.01, \mathrm{p}=0.01$ and $\mathrm{p}=0.038$, respectively). Analysing the 2 -hour ROS curves gives $\mathrm{p}=0.048$ for $\mathrm{PMN}$, $\mathrm{p}=0.035$ for PBMC and $\mathrm{p}=0.372$ for MNC. Pre-incubation of phagocytes with cytochalasin B (Cyto-B) abolished ROS production by PMN and PBMC stimulated with A. fumigatus, demonstrating that ROS production is phagocytosis dependent (figures $3 \mathrm{a}, \mathrm{b}$ ). No reduction in ROS production was observed when TLR4 or Dectin-1 were blocked on PMN and PBMC before A. fumigatus stimulation. This was similar for healthy and CF phagocytes (supplementary figure S1). To determine if the increased ROS production by CF phagocytes was a compensation mechanism necessary for adequate killing of $A$. fumigatus, the production of ROS was blocked by diphenyleneiodonium chloride (DPI) which resulted in impaired fungicidal activity. CF and healthy PBMC treated with DPI displayed reduced killing activity when compared to controls $(70.4 \% \pm 1.8 \%$ versus $80.1 \% \pm 2.4 \% \quad(\mathrm{p}=0.03)$ and $57.7 \% \pm 2.8 \%$ versus $75 \% \pm 2.3 \%$ $(\mathrm{p}=0.01$ ), respectively) (figure $4 \mathrm{a}$ ). Both CF and healthy PMN showed a $>50 \%$ drop in fungicidal activity due to DPI but did not reach statistical significance for either healthy $(18.7 \% \pm 13.5 \%$ versus $47.4 \% \pm 12.8 \%$ $(\mathrm{p}=0.13))$ or CF $(34.9 \% \pm 20.6 \%$ versus $76.7 \% \pm 5.1 \%(\mathrm{p}=0.31))$ cells (figure $4 \mathrm{~b})$. No differences were found in the relative decrease in fungicidal activity between healthy and CF phagocytes.

Increased respiratory burst in cystic fibrosis phagocytes is non-specific

In order to assess if the increased ROS production by CF phagocytes was specific to A. fumigatus, cells were stimulated with latex beads of similar size to the A. fumigatus conidia, depleted zymosan or LPS. 

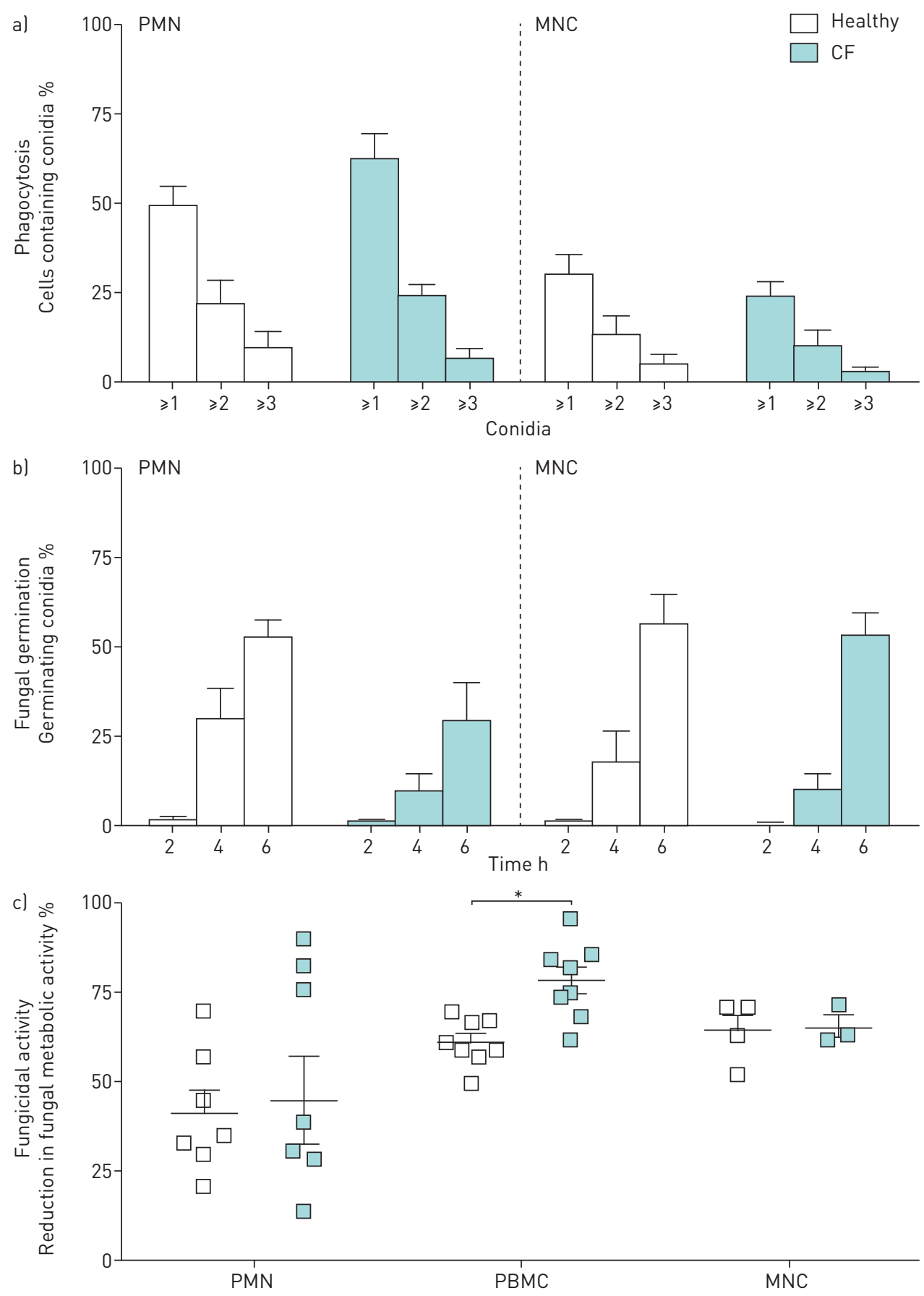

FIGURE 1 Phagocytosis and antifungal activity for phagocytes taken from healthy and CF donors. For phagocytosis (a) and fungal germination (b), polymorphonuclear cells (PMN) and monocytes (MNC) were co-incubated with FLuorescent Aspergillus REporter (FLARE) conidia in a 1:3 ratio and imaged directly with confocal microscopy. The proportion of cells containing conidia was assessed $4 \mathrm{~h}$ post-inoculation. Error bars

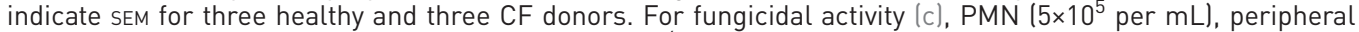
blood mononuclear cells (PBMC) and MNC $\left(2.5 \times 10^{6}\right.$ per $\left.\mathrm{mL}\right)$ were stimulated with Aspergillus fumigatus conidia $\left(2.5 \times 10^{6}\right.$ per $\left.\mathrm{mL}\right)$ for $18 \mathrm{~h}$ and fungal metabolic activity was assessed with an XTT $(2,3$-bis (2-methoxy-4-nitro-5-sulfophenyl)-2H-tetrazolium-5-carboxanilide) assay. Error bars indicate SEM for eight healthy and eight $\mathrm{CF}$ donors for PMN and PBMC and for four healthy and four CF donors for MNC. *: $p<0.05$ (Mann-Whitney U-test).

A two-fold increase in ROS production by CF PMN was observed across all conditions and similarly for CF PBMC except for LPS stimulation (figures 5a,b). However, the relative difference in ROS production between healthy and CF phagocytes remained most pronounced upon stimulation with A. fumigatus conidia. 

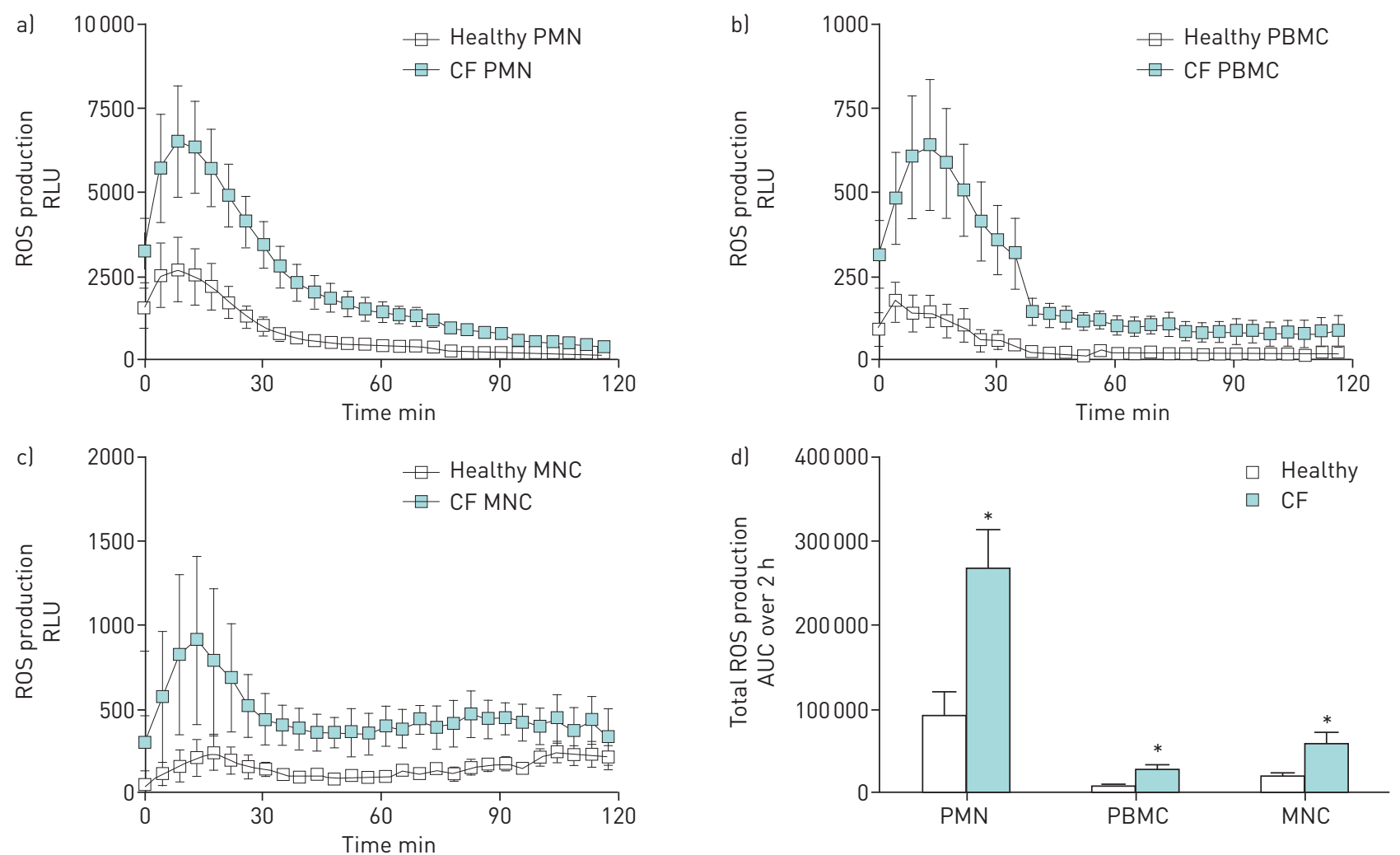

FIGURE 2 Production of reactive oxygen species (ROS) by phagocytes from healthy and CF donors. Polymorphonuclear cells (PMN) (a), peripheral blood mononuclear cells (PBMC) (b) and monocytes (MNC) (c) $\left(2.5 \times 10^{6}\right.$ per $\mathrm{mL}$ ) were stimulated with Aspergillus fumigatus conidia ( $5 \times 10^{7}$ per $\left.\mathrm{mL}\right)$ and chemiluminescence was measured directly (as relative light units (RLUs). $\mathrm{s}^{-1}$ over a 2-h period). The total ROS production (d) was calculated as the area under the curve (AUC). Error bars indicate SEM for four-to-six healthy and four-to-eight CF donors. *: p<0.05 (Mann-Whitney U-test).

To assess if peripheral CF blood phagocytes are at a higher state of activation due to soluble serum factors, cells were pre-incubated with either pooled healthy or CF serum or LPS before stimulation with A. fumigatus, beads or zymosan. Pre-incubation with LPS did not affect the ROS production by CF and healthy PMN upon stimulation with either A. fumigatus, beads or zymosan (figure 6a). In contrast, ROS production by both CF and healthy PBMC was increased approximately two-fold against depleted zymosan and three-fold against beads after pre-incubation with LPS $(p<0.05)$ (figure $6 \mathrm{~b}$ ). Under all conditions tested the ROS production of healthy phagocytes was much less than that produced by $\mathrm{CF}$
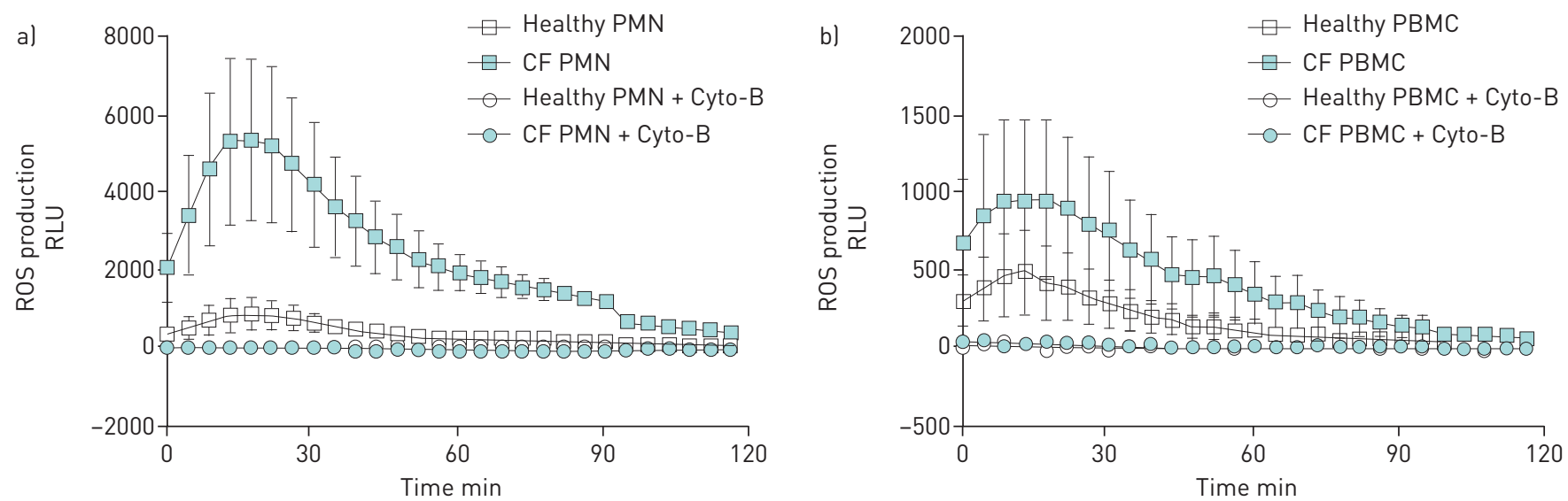

FIGURE 3 Effect of inhibition of phagocytosis on the production of reactive oxygen species (ROS) against Aspergillus fumigatus. ROS production by polymorphonuclear cells (PMN) (a) and peripheral blood mononuclear cells (PBMC) (b) $\left(2.5 \times 10^{6}\right.$ per $\left.\mathrm{mL}\right)$ is shown after pre-incubation with cytochalasin-B (Cyto-B) $\left(5 \mu \mathrm{g} \cdot \mathrm{mL}^{-1}\right)$ and stimulation with Aspergillus fumigatus conidia $\left(5 \times 10^{7}\right.$ per $\left.\mathrm{mL}\right)$. Error bars indicate SEM for four healthy and four CF donors. RLU: relative light unit. 

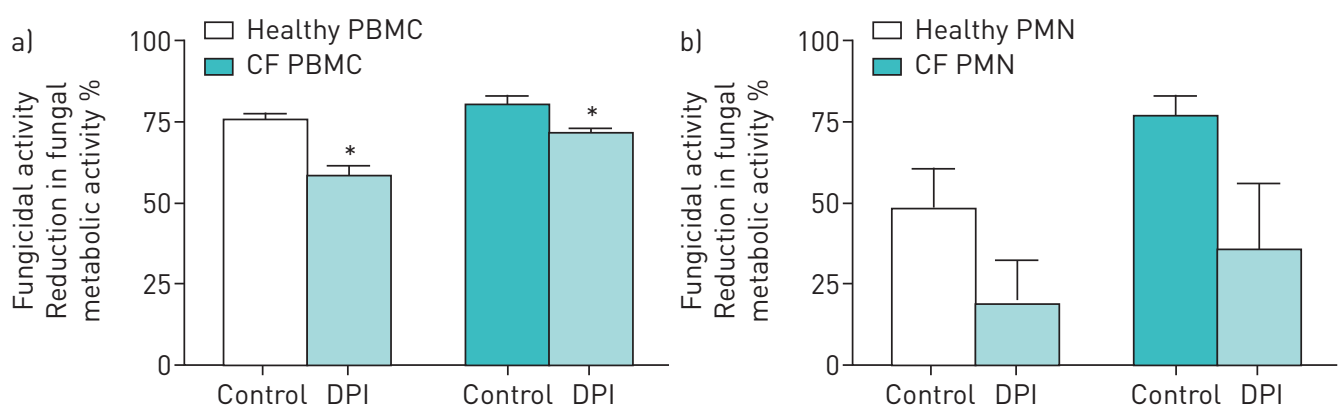

FIGURE 4 Pre-treatment of phagocytes from healthy and CF donors with diphenyleneiodonium chloride (DPI). The effect of DPI pre-incubation on the fungicidal activity of peripheral blood mononuclear cells (PBMC) (a) $\left(2.5 \times 10^{6}\right.$ per $\left.\mathrm{mL}\right)$ and polymorphonuclear cells $(P M N)$ (b) $\left(5 \times 10^{5}\right.$ per $\left.\mathrm{mL}\right)$ against Aspergillus fumigatus $\left(2.5 \times 10^{6}\right.$ per $\left.\mathrm{mL}\right)$ was assessed by XTT (2,3-bis(2-methoxy-4-nitro-5-sulfophenyl)-2H-tetrazolium5 -carboxanilidel colorimetric assay. Error bars indicate SEM for five healthy and five CF donors. *: $p<0.05$ (Mann-Whitney U-test).

phagocytes. Pre-incubating healthy PMN and PBMC with CF serum did not affect the ROS production against $A$. fumigatus conidia and beads compared to pre-incubation with healthy serum (figures $6 \mathrm{c}$ and $\mathrm{d}$ ).

\section{Cystic fibrosis polymorphonuclear cell production of reactive oxygen species against \\ Pseudomonas is not affected}

To better understand the hyperresponsive ROS production by CF PMN the cells were incubated with the exact same amount of A. fumigatus conidia, P. aeruginosa and beads of different sizes (figure 7). ROS production against $P$. aeruginosa was, remarkably, higher in absolute amounts but similar for CF and healthy PMN. A four-fold increase in ROS production was observed against A. fumigatus when comparing healthy and CF PMN ( $\mathrm{p}=0.004$ ). Furthermore, CF PMN displayed similar ROS production against $0.5 \mu \mathrm{m}$ beads compared to healthy controls; however, upon increasing bead size a strong relative increase in ROS production was observed by CF compared to healthy PMN, with a 3-fold increase against $6 \mu \mathrm{m}$ beads $(\mathrm{p}=0.0012)$.

Polymorphonuclear cell reactive oxygen species induced by A. fumigatus and clinical metrics ROS production did not differ between homozygous and heterozygous dF508 CF donors (data not shown). Increased ROS production by CF PMN was highly and significantly correlated with the number of exacerbations in the previous year ( $\mathrm{rho}=0.705, \mathrm{p}=0.007)$ and lung function. CF patients with $\mathrm{FEV} 1 \leqslant 50 \%$ predicted exhibited higher PMN ROS production than those with a FEV1 $>50 \%$ ( $\mathrm{p}=0.014$ ) (figure 8). ROS responses were comparable for patients with and without Aspergillus sensitisation (supplementary figure S2). No associations were found with positive Aspergillus IgE or IgG levels. Remarkably, ROS responses were more pronounced in those patients without Aspergillus cultured from their sputum in the previous year (4.71-fold differences in ROS production (range 2.61-7.31), $\mathrm{p}=0.03$ ).
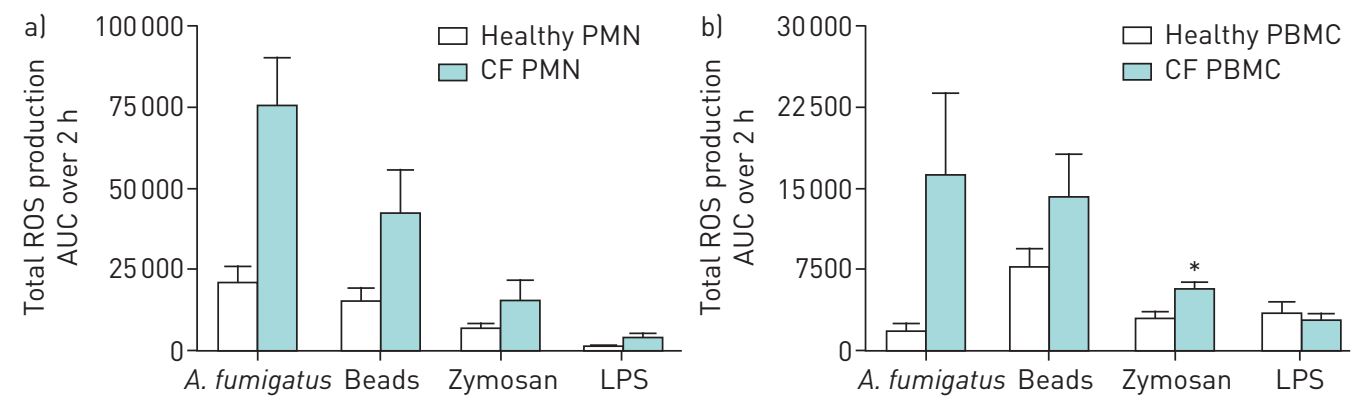

FIGURE 5 Specificity of production of reactive oxygen species (ROS) against Aspergillus fumigatus conidia by phagocytes from healthy and CF donors. Polymorphonuclear cells (PMN) (a) $\left(2.5 \times 10^{6}\right.$ per $\mathrm{mL}$ ) and peripheral blood mononuclear cells (PBMC) (b) $\left(2.5 \times 10^{6}\right.$ per $\left.\mathrm{mL}\right)$ were stimulated with A. fumigatus conidia $\left(5 \times 10^{7}\right.$ per $\mathrm{mL}), 2 \mu \mathrm{m}$ latex beads $\left(5 \times 10^{7}\right.$ per $\left.\mathrm{mL}\right)$, depleted zymosan $\left(200 \mu \mathrm{g} \cdot \mathrm{mL}^{-1}\right)$ and lipopolysaccharide (LPS) ( $50 \mathrm{ng} \cdot \mathrm{mL}^{-1}$ ). Total ROS production is shown as the area under the curve (AUC) over $2 \mathrm{~h}$ (mean \pm SEM) for five healthy and three CF donors. *: $\mathrm{p}<0.05$ (Mann-Whitney U-test). 


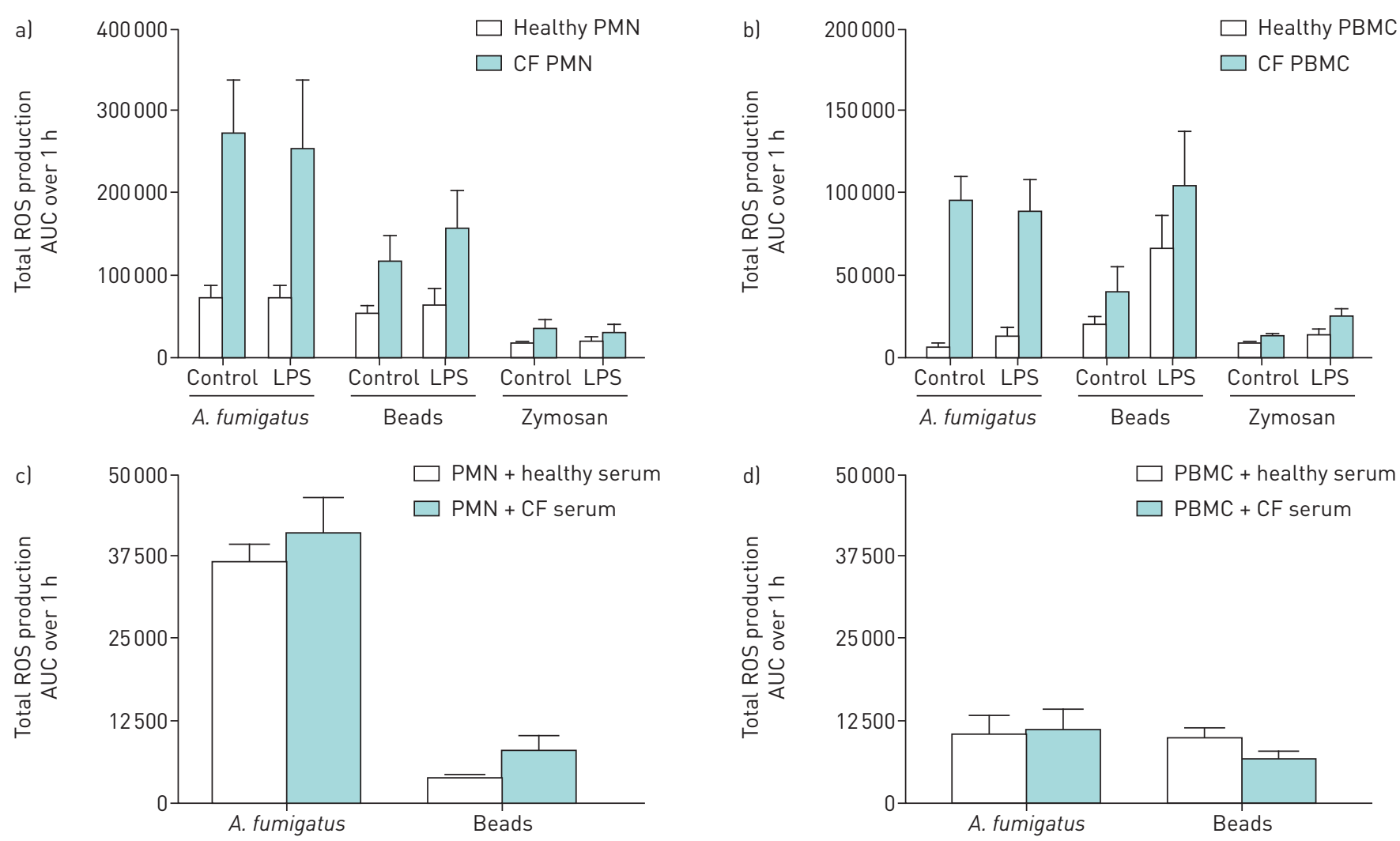

FIGURE 6 Effect of pre-treatment with lipopolysaccharide (LPS) or cystic fibrosis (CF) serum on the production of reactive oxygen species (ROS) by

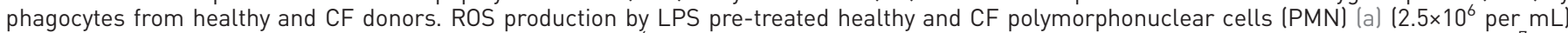
and peripheral blood mononuclear cells (PBMC) (b) $\left(2.5 \times 10^{6}\right.$ per $\left.\mathrm{mL}\right)$ was measured upon stimulation with Aspergillus fumigatus conidia (5 $\times 10^{7}$ per $\mathrm{mL})$, depleted zymosan $\left(200 \mu \mathrm{g} \cdot \mathrm{mL}^{-1}\right)$ or $2 \mu \mathrm{m}$ latex beads $\left(5 \times 10^{7}\right.$ per $\left.\mathrm{mL}\right)$. Total ROS production, as calculated by the area under the curve (AUC) of relative light units (RLUs) by chemiluminescence over $1 \mathrm{~h}$, is shown compared to non-primed controls. Error bars indicate \pm SEM for five healthy and three CF donors. Total ROS production by healthy PMN (c) $\left(2.5 \times 10^{6}\right.$ per $\left.\mathrm{mL}\right)$ and PBMC (d) $\left(2.5 \times 10^{6}\right.$ per $\left.\mathrm{mL}\right)$ incubated with $20 \%$ healthy or $\mathrm{CF}$ serum and stimulated with $A$. fumigatus conidia $\left(5 \times 10^{7}\right.$ per $\left.\mathrm{mL}\right)$ or $2 \mu \mathrm{m}$ latex beads $\left(5 \times 10^{7}\right.$ per $\left.\mathrm{mL}\right)$ was also determined. Error bars indicate \pm SEM for five healthy donors.

\section{Discussion}

The results from our study show that CF peripheral blood phagocytes are as effective as healthy cells in phagocytosis, killing and restricting germination of A. fumigatus conidia. However, CF phagocytes displayed exaggerated ROS production upon stimulation with A. fumigatus conidia compared to healthy controls. Abolishing ROS production by pre-treating the phagocytes with DPI significantly reduced fungal killing by both CF and healthy phagocytes similarly, demonstrating that the increased ROS production by CF phagocytes does not mask impaired fungal killing. ROS production was shown to be phagocytosis-dependent but not dependent on Dectin-1 and TLR4 signalling. We were able to show that the absence of a functional CFTR protein results in aberrant ROS production upon stimulation with A. fumigatus but that this is not observed for $P$. aeruginosa. Of clinical importance, the increased ROS production by CF PMN against $A$. fumigatus was highly correlated with the number of clinical exacerbations in the previous year and reduced lung function.

To our best knowledge, this is the first human study exploring the host-fungus interaction aimed at a better understanding of the role of a functional CFTR protein in innate antifungal immunity. Although associations between A. fumigatus and accelerated lung function decline in CF patients and increased hospitalisations have been reported, the clinical importance of A. fumigatus infections in CF remains less than clear and management strategies vary widely. Improving our insights into the pathophysiology of Aspergillus infections in the CF host will help to establish the clinical importance of this fungus in the progressive lung disease of $\mathrm{CF}$ and will assist in the development of targeted treatment modalities.

In this study peripheral blood phagocytes from CF patients were as effective as healthy controls in killing A. fumigatus, with even an increased fungicidal activity by CF PBMC compared to healthy cells. This was most likely due to an enhanced monocyte fraction within the CF PBMC population, as isolated CF MNC displayed no difference compared to healthy MNC. Fungicidal activity seems to be less influenced by a 


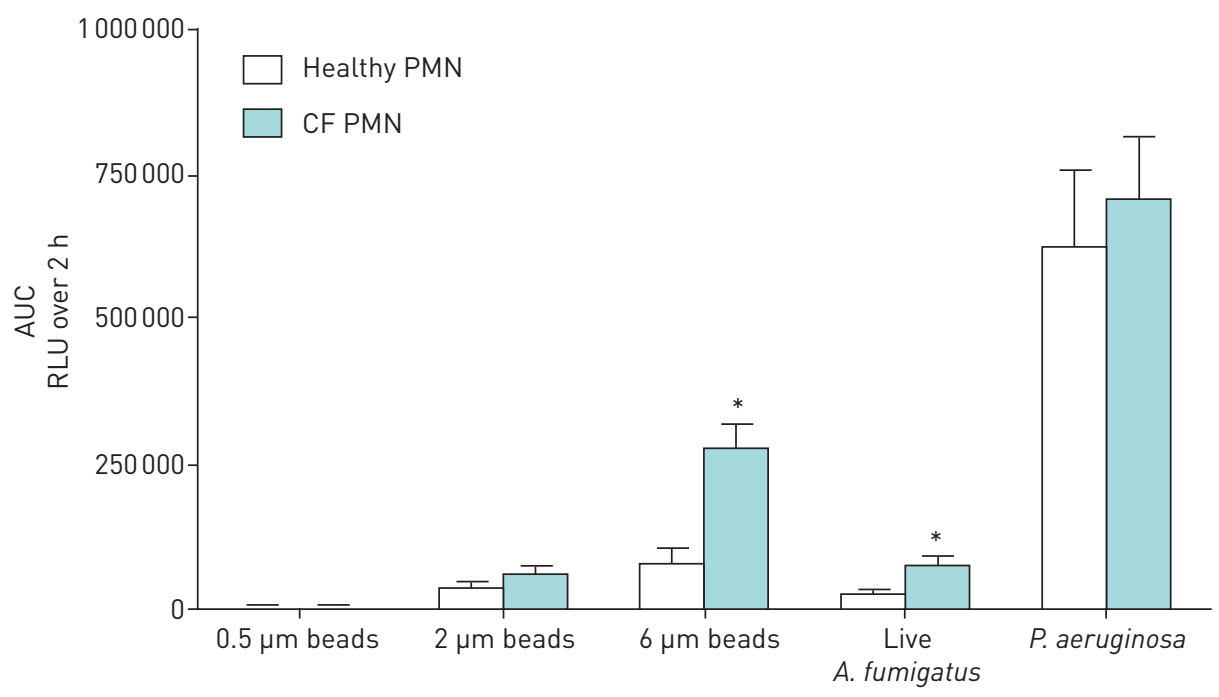

FIGURE 7 Effect on production of reactive oxygen species (ROS) by polymorphonuclear cells (PMN) from healthy and CF donors on treatment with different sizes of bead and Pseudomonas aeruginosa. PMN $\left(2.5 \times 10^{6}\right.$ per $\mathrm{mL}$ ) were stimulated with beads of different sizes $\left(0.5-6 \mu \mathrm{m} ; 5 \times 10^{7}\right.$ per $\left.\mathrm{mL}\right)$, Aspergillus fumigatus conidia and $P$. aeruginosa. Chemiluminescence was measured directly for $2 \mathrm{~h}$ and total ROS production was calculated as the area under the curve (AUC). Error bars indicate ISEM for nine healthy and $11 \mathrm{CF}$ donors. RLU: relative light unit. *: p<0.05 (Mann-Whitney U-test).

defective CFTR protein, as others have demonstrated that CF neutrophils display reduced bactericidal activity against $P$. aeruginosa [6].

Significantly increased ROS production was shown by CF PMN, PBMC and MNC upon stimulation with A. fumigatus conidia compared to healthy controls. Data from studies using specific phagocyte populations, such as CF neutrophils and monocyte-derived macrophages, have shown conflicting results regarding ROS production in vitro upon stimulation with phorbol myristate acetate (PMA), N-formylmethionyl-leucyl-phenylalanine (fMLP) or P. aeruginosa [17-20]. Interaction with fungal organisms has not been studied before and it seems likely that micro-organism-/stimulus-specific pathways do result in specific ROS responses. We did not observe a difference in ROS production against $P$. aeruginosa when comparing CF and healthy PMN although a four-fold increase in ROS was shown against $A$. fumigatus.

Dectin-1 and TLR4, which have been described as major pathogen recognition receptors (PRR) for A. fumigatus, are known to recognise conidia after uptake and binding and are associated with the initiation of an oxidative response [21-25]. However, blocking Dectin-1 and TLR4 recognition did not result in decreased ROS production by either CF or healthy phagocytes upon A. fumigatus stimulation. We

a)

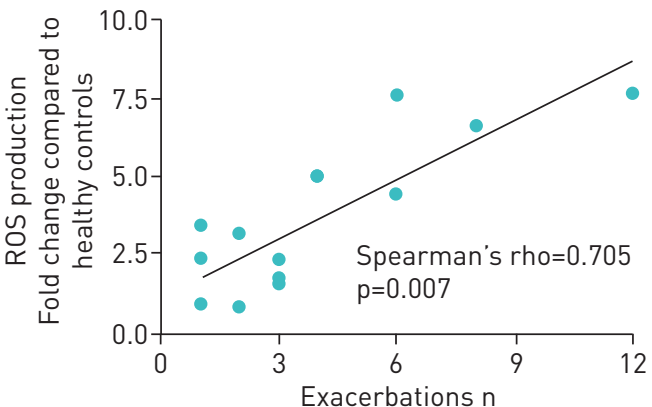

b)

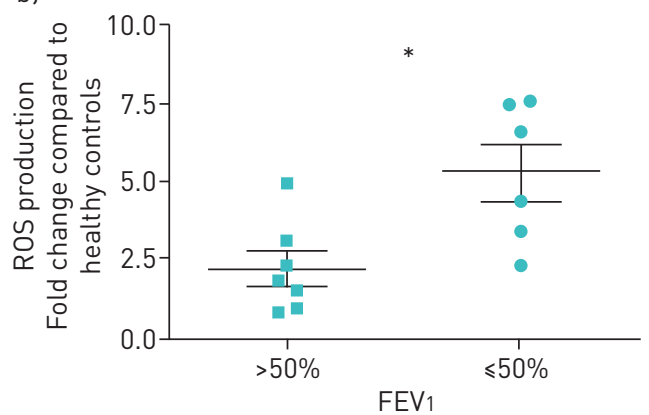

FIGURE 8 Correlation of excessive reactive oxygen species (ROS) production to clinical disease severity. ROS production against Aspergillus fumigatus by polymorphonuclear cells (PMN) from 13 cystic fibrosis (CF) patients (compared to PMN from healthy patients) was correlated with the number of exacerbations in the previous year (a). Analysis was performed using Spearman's rank correlation coefficient (Spearman's rho). In addition, the $13 \mathrm{CF}$ patients were divided into two groups based on percentage predicted forced expiratory volume in $1 \mathrm{~s}\left(\mathrm{FEV}_{1}\right)$ scores and their ROS production was compared. *: $\mathrm{p}<0.05$ (Mann-Whitney U-test). 
questioned the specificity of the increased oxidative response observed for A. fumigatus and demonstrated that $\mathrm{CF}$ phagocytes elicited a much stronger oxidative burst compared to healthy phagocytes when stimulated with beads or with specific ligands for TLR4 and Dectin-1. These results support the hypothesis reported in the literature that CF immune cells are in a general state of hyperresponsiveness $[26,27]$. Priming of healthy phagocytes with CF serum or LPS did not induce ROS responses comparable to those seen by CF phagocytes. This suggests either a specific CFTR-related form of intrinsic priming leading to a higher ROS response or a pathway of activation that takes a longer time to manifest, such as through epigenetic changes like those recently described in monocytes [28]. Aspergillus sensitisation seems not to be the explanation for the increased ROS responses as no association was found between the increased ROS responses and increased Aspergillus specific IgE or IgG levels.

It should be noted that A. fumigatus induced the highest relative increase in ROS production by $\mathrm{CF}$ phagocytes compared to healthy controls. Together with the observation that $P$. aeruginosa (a potent ROS inducer) induces similar amounts of ROS from both CF and healthy PMN, this supports the notion of a micro-organism specific induction of the oxidative response. Aspergillus conidia, at between 2 and $4 \mu \mathrm{m}$ in size, are larger than most bacteria and this amplifies dramatically once Aspergillus finds a niche and starts to germinate. The link between ROS and stimulus size has been demonstrated by PAPAYANNOPOulos et al. [29], where pathogen size directly links to the amount of ROS produced, activation of inflammatory pathways and amount of neutrophils attracted to the site of infection. When combined with our findings, e.g. positive correlation with ROS production and diameter of the beads, one explanation would be that this programme of size-dependent ROS production is affected in CF PMN leading to excessive ROS production against A. fumigatus. ROS is an essential and potent stimulator of NLRP3 and over activation of the NLRP3 inflammasome in murine CF lungs upon A. fumigatus infection has recently been shown $[30,31]$. Activation of NLRP3 in turn leads to release of interleukin-1 $($ IL-1 $\beta$ ) and using NLRP3 double knock-out mice or treatment with an IL-1 receptor antagonist has been shown to be protective against lung damage by Aspergillus [31]. The A. fumigatus induced release of excessive amounts of ROS by human CF phagocytes may well serve as a trigger for increased activation of the NLRP3 inflammasome followed by an excessive hyperinflammatory response and subsequent tissue damage in the CF lung.

Increased ROS production against $A$. fumigatus conidia by CF phagocytes may provide an explanation for the observed correlation between A. fumigatus colonisation and increased lung damage in CF [14]. The CF lung is reported to be abundant in neutrophils from early life onwards even in the absence of infectious stimuli $[32,33]$. In addition, it has been shown that bronchoalveolar lavage (BAL) fluids of CF patients contain higher levels of ROS and that these levels are correlated to the number of neutrophils present [34]. GERSUK et al. [35] showed that healthy monocyte-derived macrophages ingest and phagocytose A. fumigatus conidia effectively, while at the same time suppressing any significant ROS or pro-inflammatory cytokine production. The ability to suppress ROS production when it is not required is likely to be important because ROS are toxic compounds which attract neutrophils and mediate a multitude of inflammatory pathways [36, 37]. It is estimated that each person inhales at least 100 A. fumigatus conidia a day [38] and as such sustained and persistent ROS production can be expected in the lungs of CF patients. The significant positive correlation found in our study between A. fumigatus induced ROS production, the number of clinical exacerbations and impaired lung function support the hypothesis that Aspergillus colonisation and infection in the CF lung have an adverse impact on disease progression.

In conclusion, CF peripheral blood phagocytes are as effective as those from healthy controls regarding phagocytosis and killing or controlling the growth of A. fumigatus conidia but show hyperresponsive ROS production. The excessive amounts of ROS induced by A. fumigatus in CF neutrophils is significantly correlated to disease severity in terms of clinical exacerbations and lung function. Our data suggest that a hyperresponsive state in CF phagocytes plays a crucial role in the hyperinflammatory response upon exposure to A. fumigatus. New interventions to prevent fungal induced ROS production need to be explored, such as early treatment of Aspergillus infection or targeting the defective molecular pathway underlying these aberrant responses.

Acknowledgements: We would like to thank the CF patients for their participation and a special thank you goes to CF nurses Karen Griffiths and Sandra Steele (Aberdeen Royal Infirmary, Aberdeen, UK) for their invaluable contribution to this study.

Author contributions: A. Warris and G. Devereaux conceived and designed this study; S.F. Brunel and J.A. Willment designed and performed the study; A. Warris, G. Devereaux, S.F. Brunel and G.D. Brown analysed and interpreted the data; and all authors drafted the manuscript for intellectual content.

Conflict of interest: A. Warris reports receiving grants and personal fees from Gilead, and personal fees from Basilea, outside the submitted work. 
Support statement: A. Warris, G.D. Brown, S.F. Brunel and J.A. Willment were supported by the Wellcome Trust Strategic Award (grant 097377) and the MRC Centre for Medical Mycology (grant MR/N006364/1) at the University of Aberdeen. A. Warris and S.F. Brunel were also supported by the Chloe Fund. Funding information for this article has been deposited with the Crossref Funder Registry.

\section{References}

Farrell PM. The prevalence of cystic fibrosis in the European Union. J Cyst Fibros 2008; 7: 450-453.

Cohen TS, Prince A. Cystic fibrosis: a mucosal immunodeficiency syndrome. Nat Med 2012; 18: 509-519.

Zhou Y, Song K, Painter RG, et al. Cystic fibrosis transmembrane conductance regulator recruitment to phagosomes in neutrophils. J Innate Imm 2013; 5: 219-230.

4 Johansson J, Vezzalini M, Verzè G, et al. Detection of CFTR protein in human leukocytes by flow cytometry. Cytometry A 2014; 85: 611-620.

5 Rieber N, Hector A, Carevic M, et al. Current concepts of immune dysregulation in cystic fibrosis. Int J Biochem Cell Biol 2014; 52: 108-112.

6 Painter RG, Bonvillain RW, Valentine VG, et al. The role of chloride anion and CFTR in killing of Pseudomonas aeruginosa by normal and CF neutrophils. J Leukoc Biol 2008; 83: 1345-1353.

7 Del Porto P, Cifani N, Guarnieri S, et al. Dysfunctional CFTR alters the bactericidal activity of human macrophages against Pseudomonas aeruginosa. PLoS One 2011; 6: e19970.

8 Van de Weert-van Leeuwen PB, Van Meegen MA, Speirs JJ, et al. Optimal complement mediated phagocytosis of Pseudomonas aeruginosa by monocytes is cystic fibrosis transmembrane conductance regulator-dependent. Am J Respir Cell Mol Biol 2013; 49: 463-470.

9 Assani K, Tazi MF, Amer AO, et al. IFN- $\gamma$ stimulates autophagy-mediated clearance of Burkholderia cenocepacia in human cystic fibrosis macrophages. PLoS One 2014; 9: e96681.

10 Warris A. The biology of pulmonary Aspergillus infections. J Infect 2014; 69: S36-S41

11 Pihet M, Carrere J, Cimon B, et al. Occurrence and relevance of filamentous fungi in respiratory secretions of patients with cystic fibrosis-a review. Med Mycol 2009; 47: 387-397.

12 Armstead J, Morris J, Denning DW. Multi-country estimate of different manifestations of aspergillosis in cystic fibrosis. PLOS ONE 2014; 9: e98502.

13 de Vrankrijker AM, van der Ent CK, van Berkhout FT, et al. Aspergillus fumigatus colonization in cystic fibrosis: implications for lung function? Clin Microbiol Infect 2011; 17: 1381-1386.

14 Amin R, Dupuis A, Aaron SD, et al. The effect of chronic infection with Aspergillus fumigatus on lung function and hospitalization in patients with cystic fibrosis. Chest 2009; 137: 171-176.

15 Jhingran A, Mar KB, Kumasaka DK, et al. Tracing conidial fate and measuring host cell antifungal activity using a reporter of microbial viability in the lung. Cell Rep 2012; 2: 1762-1773.

16 Henriet SSV, Hermans PWM, Verweij PE, et al. Human leukocytes kill Aspergillus nidulans by reactive oxygen species independent mechanisms. Infect Immun 2011; 79: 767-773.

17 Houston N, Stewart N, Smith DS, et al. Sputum neutrophils in cystic fibrosis patients display a reduced respiratory burst. J Cyst Fibros 2013; 12: 352-362.

18 Cifani N, Pompili B, Anile M, et al. Reactive-oxygen-species mediated P. aeruginosa killing is functional in human cystic fibrosis macrophages. PLoS One 2013; 8: e71717.

19 McKeon DJ, Cadwallader KA, Idris S, et al. Cystic fibrosis neutrophils have normal intrinsic reactive oxygen species generation. Eur Respir J 2010; 35: 1264-1272.

20 Vaisman N1, Kerasin E, Hahn T, et al. Increased neutrophil chemiluminescence production in patients with cystic fibrosis. Metabolism 1994; 43: 719-722.

21 Faro-Trindade I, Willment JA, Kerrigan AM, et al. Characterisation of innate fungal recognition in the lung. PLoS One 2012; 7: e35675.

22 Taylor PR, Brown GD, Reid DM, et al. The $\beta$-glucan receptor, dectin-1, is predominantly expressed on the surface of cells of the monocyte/macrophage and neutrophil lineages. J Immunol 2002; 169: 3876-3882.

23 Metz AE, Horn D, Schoeb TR, et al. Requisite role for the dectin-1 $\beta$-glucan receptor in pulmonary defense against Aspergillus fumigatus. J Immunol 2009; 182: 4938-4946.

24 Netea MG, Warris A, Van der Meer JW, et al. Aspergillus fumigatus evades immune recognition during germination through loss of toll like receptor-4-mediated signal transduction. J Infect Dis 2003; 188: 320-326.

25 Ryan KA, Smith MF Jr, Sanders MK, et al. Reactive oxygen and nitrogen species differentially regulate Toll-like receptor 4-mediated activation of NF-kappa B and interleukin-8 expression. Infect Immun 2004; 72: 2123-2130.

26 Koller DY, Urbanek R, Götz M. Increased degranulation of eosinophil and neutrophil granulocytes in cystic fibrosis. Am J Respir Crit Care Med 1995; 152: 629-633.

27 Taggart C, Coakley RJ, Greally P, et al. Increased elastase release by CF neutrophils is mediated by tumor necrosis factor- $\alpha$ and interleukin-8. Am J Physiol Lung Cell Mol Physiol 2000; 278: L33-L41.

28 Saeed S, Quintin J, Kerstens HH, et al. Epigenetic programming of monocyte-to-macrophage differentiation and trained innate immunity. Science 2014; 345: 1251086

29 Warnatsch A, Tsourouktsoglou TD, Branzk N, et al. Reactive oxygen species localization programs inflammation to clear microbes of different size. Immunity 2017; 46: 421-432.

30 Bauernfeind F, Bartok A, Rieger A, et al. Reactive oxygen species inhibitors block priming, but not activation of the NLRP3 inflammasome. J Immunol 2011; 187: 613-617.

31 Iannitti RG, Napolioni V, Oikonomou V, et al. IL-1 receptor antagonist ameliorates inflammasome-dependent inflammation in murine and human cystic fibrosis. Nat Commun 2016; 7: 10791.

32 Verhaeghe C, Delbecque K, de Leval L, et al. Early inflammation in the airways of a cystic fibrosis foetus. J Cyst Fibros 2007; 6: 304-308

33 Tirouvanziam R, de Bentzmann S, Hubeau C, et al. Inflammation and infection in naïve human cystic fibrosis airway grafts. Am J Respir Cell Mol Biol 2000; 23: 121-127.

34 Starosta V, Rietschel E, Paul K, et al. Oxidative changes of bronchoalveolar proteins in cystic fibrosis. Chest 2006; 129: 431-437. 
35 Gersuk GM, Underhill DM, Zhu L, et al. Dectin-1 and TLRs permit macrophages to distinguish between different Aspergillus fumigatus cellular states. J Immunol 2006; 176: 3717-3724.

36 Renkawitz J, Sixt M. A radical break: restraining neutrophil migration. Dev Cell 2016; 38: 448-450.

37 Thannickal VJ, Fanburg BL. Reactive oxygen species in cell signaling. Am J Physiol Lung Cell Mol Physiol 2000; 279: L1005-L1028.

38 Latgé J. Aspergillus fumigatus and aspergillosis. Clin Microbiol Rev 1999; 12: 310-350. 